\title{
Programa de Ginástica Laboral e a Redução de Atestados Médicos
}

\section{LABOR GYMNASTICS PROGRAM AND REDUCTION OF MEDICAL CERTIFICATES}

\section{Rafael Cunha Laux', Paulo Pagliari², João Viannei Effting Junior ${ }^{3}$, Sara Teresinha Corazza ${ }^{4}$}

1. Mestrando do Curso de Pós-Graduação em Educação Física (UFSM) Santa Maria/RS, Bolsista Capes, Especialista em Personal Training (UNOESC) Chapecó/SC.

2. Mestre em Educação - área de concentração Educação Física e Saúde pelo Centro Universitário Diocesano do Sudoeste do Paraná, Coordenador do curso de Educação Física da UNOESC - Campus de Chapecó.

3. Mestre em Engenharia Biomédica pela Universidade de Mogi das Cruzes. Graduando em Medicina pela UNOCHAPECÓ.

4. Doutora em Ciência do Movimento Humano pela Universidade Federal de Santa Maria, Professora do CEFD/UFSM.

\section{RESUMO}

Estudos recentes têm apontado a ginástica laboral como uma importante ferramenta para amenizar alguns malefícios ocasionados pelo ritmo de trabalho da vida moderna. Portanto, o presente estudo objetivou analisar a influência de um programa de ginástica laboral (PGL) na redução da quantidade de atestados de uma empresa de tratamento de resíduos industriais da cidade de Chapecó-SC. A amostra foi composta por 31 sujeitos, sendo 22 do sexo masculino e 9 do sexo feminino com idade média de $32,48 \pm 8,28$. Todos os dados foram coletados a partir dos prontuários dos funcionários. 0 PGL foi desenvolvido duas vezes na semana durante 12 meses contínuos. Os resultados apontam uma diminuição na quantidade total de atestados $(51,52 \%)$, por doenças sistêmicas $(43,48 \%)$ e por doenças osteomusculares $(55,56 \%)$ no período de desenvolvimento do PGL. Conclui-se portanto, que com a diminuição no número de atestados, houve também melhoria na saúde e qualidade de vida de seus praticantes.

(Laux R, Pagliari P, Viannei J, Corazza S, 2016. Programa de Ginástica Laboral e a Redução de Atestados Médicos. Cienc Trab. MayAgo; 18 [56]: 130-133).

Palavras chaves: SAÚDE DO TRABALHADOR, ATESTADO DE SAÚDE, QUALIDADE DE VIDA, TRABALHADORES.

\section{ABSTRACT}

Recent studies have pointed the labor gymnastics as an important tool to alleviate some caused by the working in the modern life. Therefore, the present study aimed to analyze the influence of a labor gymnastics program (LGP) in reduction the amount of certificates of industrial in the city of Chapecó-SC. The sample consisted of 31 subjects, 22 males and 9 females with an average age of 32.48 \pm 8.28 . All data were collected from the medical records of employees. The LGP was developed two times a week for 12 months continuous. The results show a decrease in the total amount of certificates $(51.52 \%)$, for systemic diseases $(43.48 \%)$ and musculoskeletal diseases $(55.56 \%)$ in the period of development on the LGP. In conclusion, with the decrease in the number of certificates, there was also improvement in health and quality of life of its practitioners.

Keywords: OCCUPATIONAL HEALTH, HEALTH CERTIFICATE, QUALITY OF LIFE, WORKERS.

\section{INTRODUÇ̃̃O}

A modernidade vem transformando o dia-a-dia das pessoas, aumentando as horas de trabalho e diminuindo o tempo de ócio e lazer, fazendo com que esse excesso de trabalho muitas vezes se transforme em doenças físicas e/ou mentais. ${ }^{1,2}$ Essa mudança no cenário do trabalho ocasionou o aumento das doenças ocupacionais, não somente a lesão por esforço repetitivo (LER) e o distúrbio

Correspondencia / Correspondence:

Rafael Cunha Laux

Universidade do Oeste de Santa Catarina - UNOESC, Campus de Chapecó

Av. Nereu Ramos, 3777-D - Bairro Seminário, Chapecó-SC, Brasil

CEP:898113-000.

Tel.: (049) 33192643

e-mail: rafael-laux@hotmail.com

Recibido: 06 de Marzo de 2016 / Aceptado: 28 de Mayo de 2016 osteomusculares relacionados ao trabalho (DORT), mas as doenças de origem psicológica, frutos do stress. ${ }^{3}$

As doenças osteomusculares e as mentais são dois grupos de patologias que trazem maior incapacidade e afastamento do trabalho. ${ }^{4}$ Os gastos originários de doenças ocupacionais são raramente contabilizados, mesmo em países que investem na prevenção. Esses valores são estimados em $4 \%$ do produto interno bruto (PIB) para os países desenvolvidos e 10\% do PIB para países em desenvolvimento, como é o caso do Brasil. ${ }^{5}$

Percebendo a necessidade de diminuir ou prevenir as doenças ocupacionais, as empresas estão investindo na prática de exercícios físicos no próprio local de trabalho. ${ }^{1,2,6}$ Com o intuito de promover a saúde do trabalhador com a prática de exercícios físicos surge o Programa de Ginástica Laboral (PGL), também conhecido internacionalmente como Intervenções de Exercício Físico no Ambiente de Trabalho (Workplace Physical Activity Intervention), que conta com programa de exercícios físicos específicos para compensar os grupos musculares mais exigidos durante as atividades laborais ${ }^{6}$, um exercício físico-motor que pode compensar desgastes e auxiliar em prevenções. 0 programa 
pode ter amplos objetivos, como a promoção do autoconhecimento, da autoestima, e possiveis melhorias do convívio social na empresa. ${ }^{2,7}$

Os principais benefícios para a empresas que contratam profissionais para a realização do PGL são o aumento da produtividade, diminuição da incidência das doenças ocupacionais e a diminuição das despesas médicas. Para os funcionários podemos destacar como benefícios a melhora na autoestima, melhora no relacionamento interpessoal, a redução de dores, redução de stress, diminuição da fadiga e melhora na saúde física, metal e emocional. ${ }^{8}$ Além dos benefícios tradicionalmente conhecidos na literatura, a ginástica laboral influencia positivamente outras variáveis que podem interferir na saúde do trabalhador, como é o caso da coordenação motora global e tempo de reação. ${ }^{9}$

Para que a empresa e o funcionário obtenham todos os benefícios do PGL, é necessário uma avaliação, planejamento e aplicação por profissional habilitado ${ }^{2,6}$, incidindo positivamente nas adesões dos funcionários. ${ }^{10}$ Outro fato relatado sobre a prática orientada de exercícios físicos no ambiente de trabalho é a predisposição desses funcionários em praticar exercícios fora do ambiente de trabalho, contribuindo positivamente para sua saúde. ${ }^{11}$

Nessa perspectiva o objetivo desse estudo foi analisar se um programa de ginástica laboral (PGL) influenciou na redução da quantidade de atestados de uma empresa de tratamento de resíduos industriais da cidade de Chapecó-SC.

\section{MATERIAL E MÉTODO}

Esta pesquisa classificou-se quanto aos objetivos como descritivocomparativo $^{12}$, e com base nos procedimentos técnicos a pesquisa se enquadra como documental. ${ }^{13}$ Nessa pesquisa documental foram utilizados documentos de "primeira mão", segundo Gil ${ }^{13}$ esses documentos não recebem nenhum tipo de tratamento analítico, como documentos conservados em arquivos de empresas ou órgãos públicos. 0 projeto foi aprovado pelo Comitê de Ética em Pesquisa da Universidade do Oeste de Santa Catarina pelo CAAE n ${ }^{\circ}$ 42803815.5.0000.5367, e dispensa Termo de Consentimento Livre e Esclarecido conforme prevê a resolução 196/96 do Conselho Nacional de Saúde.

A população deste estudo envolveu 113 funcionários entre 17 e 53 anos de uma empresa de tratamento de resíduos industriais da cidade de Chapecó-SC. Os funcionários eram oriundos dos setores administrativo ou de produção. No setor administrativo os sujeitos passavam a maior parte do tempo sentados e em frente a computadores. Já na produção os funcionários apresentam atividades mais braçais, predominantemente em pé, com pequenos deslocamentos e movimentos de agachamento levantando diferentes cargas.

Para participar do estudo os sujeitos precisavam atender aos seguintes critérios: 1) estar contratado pela empresa no período do estudo (julho 2012 a junho 2014); e 2) ter frequência mínima de 75\% nas aulas de Ginástica Laboral ministradas entre julho de 2013 e junho de 2014.

0 instrumento utilizado foi o prontuário do funcionário, neles foram coletados os seguintes dados: a) dados que caracterizem a amostra; b) quantidade, motivo e tempo dos afastamentos por atestado médico; c) frequência e características técnicas do programa de ginástica laboral, entre os meses de julho de 2013 e junho de 2014. Os dados utilizados para caracterizar a amostra foram a idade e 0 sexo. No arquivo foi verificado se a data de admissão do funcionário ocorreu antes de julho de 2012. Para a análise dos atestados médicos foi selecionado apenas os relacionados a doença do funcionário, a tratamento médico e a tratamento cirúrgico. E excluído os atestados médicos relacionados ao acompanhamento de familiar em consulta ou similar; consultas médicas agendadas; consulta e tratamento odontológico; exames médicos e similares; gravidez; atestados sem o motivo do afastamento ou data; cirurgias plásticas; e acidentes de trabalho. Nesse período a empresa não desenvolveu nenhuma campanha ou intervenção para melhorar a qualidade de vida, para não interferir nos resultados do PGL.

O PGL disponibilizado na empresa foi realizado por profissional capacitado (formado em Educação Física e registrado no Conselho Regional de Educação Física - CREF/SC), e desenvolveu-se com aulas de 10 minutos, 2 vezes por semana durante 12 meses. Cada aula incluiu parte inicial (4 min), parte principal (4 min) e parte final (2 min). A parte inicial teve ênfase no aumento dos batimentos cardíacos com atividades globais de pequenos deslocamentos e de coordenação de multimembros. A segunda parte (principal) foi composta por exercícios de flexionamento para os membros superiores e inferiores, e/ou reforço muscular com exercícios isométricos e dinâmicos, específicos para função dos trabalhadores, considerando os setores já mencionados. A parte final teve atividades de descontração, alongamento e de volta à calma (QUADRO 1). As aulas foram elaboradas após análise dos postos de trabalho e funções profissionais específicas. A ginástica laboral teve como característica a ginástica compensatória. ${ }^{2}$

Quadro 1.

Atividades do Programa de Ginástica Laboral.

\begin{tabular}{|c|c|c|c|}
\hline & $\begin{array}{l}\text { Parte Inicial } \\
\text { (4minutos) }\end{array}$ & $\begin{array}{l}\text { Parte Principal } \\
\text { (4minutos) }\end{array}$ & $\begin{array}{l}\text { Parte Final } \\
\text { (2 minutos) }\end{array}$ \\
\hline ATIVIDADES & $\begin{array}{l}\text { Atividades recreativas, } \\
\text { utilizando ritmo, } \\
\text { coordenação } \\
\text { motora fina, atenção, } \\
\text { rapidez de } \\
\text { raciocínio. }\end{array}$ & $\begin{array}{l}\text { Exercícios de flexionamento } \\
\text { e de fortalecimento } \\
\text { státicos e dinâmicos } \\
\text { para todos grupos } \\
\text { musculares, enfatizando } \\
\text { os mais utilizados na } \\
\text { jornada de trabalho. }\end{array}$ & $\begin{array}{l}\text { Exercícios de } \\
\text { respiração, } \\
\text { dinâmicas de grupo, } \\
\text { alongamento e } \\
\text { exercícios de } \\
\text { concentração. }\end{array}$ \\
\hline $\begin{array}{l}\text { MATERIAIS } \\
\text { UTILIZADOS }\end{array}$ & $\begin{array}{l}\text { Bolinhas de borracha, } \\
\text { bastões, arcos, jornal, } \\
\text { bola de vôlei, músicas. }\end{array}$ & $\begin{array}{l}\text { Colchonetes, bastões, } \\
\text { arcos, bolinhas de borrachas, } \\
\text { músicas. }\end{array}$ & $\begin{array}{l}\text { Músicas calmas, } \\
\text { colchonetes. }\end{array}$ \\
\hline
\end{tabular}

Para o tratamento dos dados, por contarmos com um reduzido número de sujeitos, foi somente utilizado estatística descritiva, através da média, desvio padrão, mínimo, máximo e frequência.

\section{RESULTADOS}

A amostra foi composta por 31 sujeitos, sendo 22 do sexo masculino e 9 do sexo feminino com idade média de 32,48 $\pm 8,28$ em junho de 2014 , conforme a tabela 1. Entre os sujeitos 14 do sexo masculino e 4 do sexo feminino não houve nenhum registro de afastamento por atestado médico no período do estudo.

Tabela 1.

Características da amostra.

\begin{tabular}{lllll} 
& $\begin{array}{l}\text { Média da } \\
\text { Idade } \\
\text { (Jun/2014) }\end{array}$ & $\begin{array}{l}\text { Desvio Padrão } \\
\text { da Idade } \\
\text { (Jun/2014) }\end{array}$ & $\begin{array}{l}\text { Mínimo da } \\
\text { Idade } \\
\text { (Jun/2014) }\end{array}$ & $\begin{array}{l}\text { Máximo da } \\
\text { Idade } \\
\text { (Jun/2014) }\end{array}$ \\
\hline Masculino $(n=22)$ & 32,36 & 8,20 & 19 & 51 \\
Feminino $(n=9)$ & 32,78 & 9,23 & 20 & 50 \\
Total $(n=31)$ & 32,48 & 8,28 & 19 & 51
\end{tabular}


A figura 1 apresenta a comparação entre a quantidade de afastamento por atestados médicos no período sem ginástica laboral (junho de 2012 a julho de 2013), e o período com ginástica laboral (junho de 2013 a julho de 2014). Os afastamentos por atestados médicos foram separados em dois grupos, os relacionados as doenças sistêmicas (excluindo-se as doenças sexualmente transmissiveis), e as relacionadas a doenças osteomusculares. As doenças sistêmicas relatadas nos atestados médicos foram varizes nos membros inferiores, reumatismo não especificado, dores não especificadas no abdômen e hipertensão arterial. Já nas doenças osteomusculares os atestados foram por causa de hérnia inguinal e lombalgias.

Figura 1.

Comparação entre os afastamentos por atestados médios no período com e sem ginástica laboral.

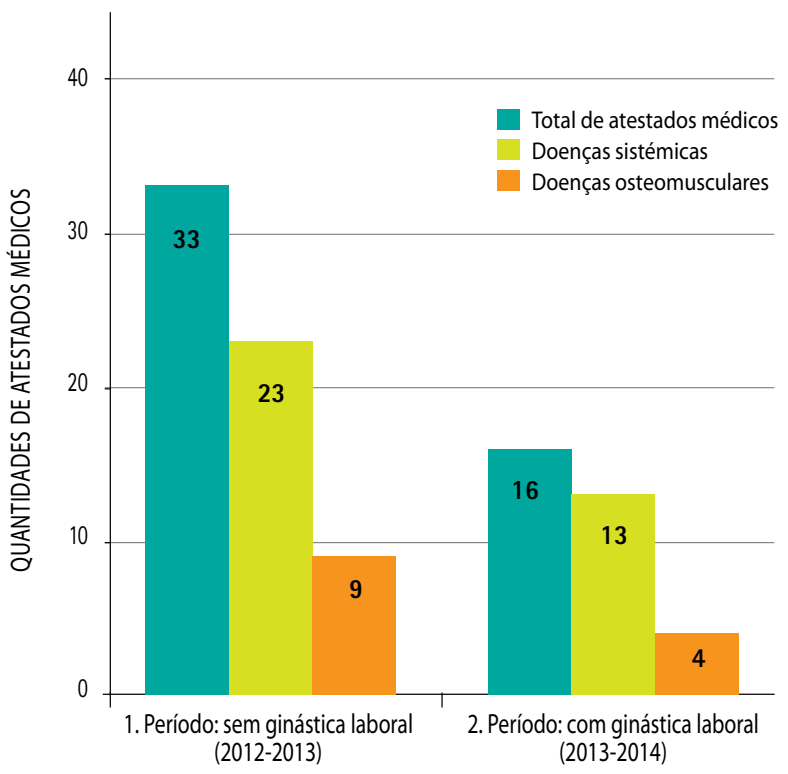

Observa-se que no período sem ginástica laboral os afastamentos por atestados médicos representaram 33 atestados, sendo que no período com ginástica laboral foram 16, uma redução de 51,52\%. Nas doenças sistêmicas observa-se uma diminuição de $43,48 \%$ dos atestados após a implantação do PGL. Ao analisar as doenças osteomusculares verificamos que o primeiro período apresentou 9 atestados, e no segundo houve uma redução de 55,56\%, passando para 4 atestados.

\section{DISCUSSÃO}

A busca pelo aumento da produtividade e a diminuição dos afastamentos por atestados médicos faz da ginástica laboral uma aliada. ${ }^{2,14}$ Entretanto, são poucas pesquisas que exploram as causas da quantidade de afastamento por atestados médicos. ${ }^{15}$

Os resultados encontrados nesse estudo corroboram com os evidenciados por Silveira ${ }^{16}$, onde teve uma diminuição da quantidade de absenteísmo em ambos os sexos durante a aplicação do PGL. Essa tendência de diminuição de absenteísmo também ocorreu na pesquisa de Reis ${ }^{17}$ onde evidenciou-se uma queda de 6 afastamentos durante o período da intervenção com exercícios de aquecimento e alongamento. No estudo de Martinez e Matiello Júnior ${ }^{18}$ os indivíduos relatam uma melhora na sua condição de saúde física, o que pode resultar numa menor quantidade de afastamento por doenças.
Coelho e Burini ${ }^{19}$ relatam inúmeros estudos epidemiológicos que relacionam o nível de prática de exercício físico com a diminuição das doenças, porém os mecanismos relacionados a esse fato não está totalmente compreendido, não existindo um consenso sobre a quantidade necessária de exercício para esse benefício. Bielemann, Knuth e Hallal $^{20}$ ressaltam o exercício físico como meio de prevenção de doenças, afirmando que sua prática diminui doenças crônicas, gerando uma economia nos gastos públicos da saúde. Observa-se que como nos estudos de Coelho e Burini ${ }^{19}$ e Bielemann, Knuth e Hallal ${ }^{20}$ os resultados apresentados na presente pesquisa apontam uma diminuição das doenças sistêmicas, mesmo com número e tempo de aula reduzido.

Reis, Moro e Contijo ${ }^{21}$ em seu estudo observaram uma diminuição dos atestados médicos, que tratavam especificamente sobre lombalgias, após a implantação do programa de ginástica laboral. Fato que corrobora com os resultados encontrados nesse estudo, onde verificou-se a queda de cerca de 55,56\% na quantidade de atestados por doenças osteomusculares.

Outro fator que pode, talvez, explicar a diminuição dos atestados por doenças osteomusculares está evidenciado na pesquisa de Candotti, Stroschein e Noll $^{22}$ quando relatam a diminuição da intensidade e frequência das dores nos grupos musculares envolvidos no trabalho diário, após três meses de intervenção com o PGL. A diminuição das dores lombares foi apontada no estudo de Reis, Moro, Contijo ${ }^{21}$, sendo que essas tiveram um decréscimo de $90 \%$ dos casos durante a após a aplicação do PGL.

A importância de prevenir as doenças osteomusculares como a LER/ DORT vai muito além de aspectos físicos, podendo causar repercussões na saúde mental do funcionário e desencadear transtornos mentais. ${ }^{23}$ Picoloto e Silveira ${ }^{24}$, e Szeto e $\mathrm{Lam}^{25}$ destacam a grande prevalência de sintomas osteomusculares em trabalhadores e sua relação com idade, sexo, ocupação e escolaridade.

Os cofres públicos e privados também detectam os prejuizos causados pelos afastamentos dos funcionários por causa de atestados médicos. Segundo Brasil ${ }^{3}$ não existe pesquisa com os gastos totais resultantes das doenças e acidentes de trabalhos, mas estima-se que esses valores chegam a $R$ \$ 12,5 bilhões anuais para as empresas e em mais de $R$ \$ 20 bilhões anuais para os contribuintes. Santana et $\mathrm{al}^{5}$ identificaram 257.645 benefícios concedidos pelo INSS para trabalhadores no estado da Bahia no ano 2000, onde 11,7\% referiam-se a problemas de saúde ocupacional, enquanto $7,3 \%$ aos acidentes ocupacionais (causas externas, lesões e envenenamentos).

Ao analisar a importância de um programa de ginástica laboral influenciar na redução da quantidade de atestados médicos e consequentes afastamentos em uma empresa de tratamento de resíduos industrias da cidade de Chapecó-SC, observou-se uma redução em números absolutos de 51,52\% nesses atestados, tanto por doenças sistêmicas, quanto por doenças osteomusculares.

Fato que corrobora com a literatura, em que enfatiza a importância da ginástica laboral como meio de prevenção de doenças, diminuição de absenteísmo, melhora da relação interpessoal, e consequentemente uma diminuição de custos relacionados ao afastamento do funcionário de sua função.

\section{Agradecimentos}

Agradecimento especial ao Curso de Pós-graduação lato sensu em Personal Training da Universidade do Oeste de Santa Catarina Campus de Chapecó, que possibilitou a produção desse trabalho, tendo em vista que esse artigo é fruto da pesquisa desenvolvida como requisito parcial para obtenção do título de Especialista em Personal Training. 
1. Mezzomo SP, Contreira AR, Corazza ST. Os efeitos da ginástica laboral sobre as habilidades básicas de funcionários de setores administrativos. $\mathrm{R}$ Bras $\mathrm{Ci}$ Saúde. 2010;8(25):6-13.

2. Figueiredo $F$, Mont'alvão $C$. Ginástica laboral e ergonomia. $2^{2}$ ed. Rio de Janeiro: Sprint; 2008.

3. Brasil. Ministério da Saúde. Doenças relacionadas ao trabalho: manual de procedimentos para os serviços de saúde. Brasília: Ministério da Saúde; 2001.

4. Barbosa-Branco A, Albuquerque-Oliveira PR. Prevalência de incapacidade para o trabalho no setor saúde no Brasil, 2004. Tempus Actas de Saúde Colet. $2011 ; 5(1): 235-250$.

5. Santana VS, Araujo-Filho JB, Albuquerque-Oliveira PR, Barbosa-Branco A. Acidentes de trabalho: custos previdenciários e dias de trabalho perdidos. Rev Saúde Pública. 2006; 40(6):1004-1012.

6. Pereira CCDA. Excelência técnica dos programas de ginástica laboral: uma abordagem didático-pedagógico. São Paulo: Phorte; 2013.

7. Poletto SS. Avaliação e implantação de programas de ginástica laboral, implicações metodológicas [dissertação de Mestrado]. Porto Alegre-RS: UFRGS; 2002.

8. Militão AG. A influência da ginástica laboral para a saúde dos trabalhadores e sua relação com os profissionais que a orientam [dissertação de Mestrado]. Florianópolis-SC: UFSC; 2001.

9. Mezzomo SP, Cardozo PL, Katzer Jl, Santos DL, Corazza ST. A influência da ginástica laboral na coordenação motora global e no tempo de reação de condutores de autocarro. Motricidade. 2014;10(4):27-34.

10. Soares $R G$, Assunção $A A$, Lima FPA. A baixa adesão ao programa de ginástica laboral: buscando elementos do trabalho para entender o problema. Revis bras saúde ocup. 2006;31(114):149-160.

11. Dias $A G$, Silva IAS, Silva VF, Beltrão FB. A contribuição de um programa de ginástica laboral para a aderência ao exercício físico fora da jornada de trabalho. Fit Perf J. 2006; 5(5):325-332.

12. Thomas JR, Nelson JK, Silverman S. Métodos de pesquisa em atividade física. $5^{a}$ ed. Porto Alegre: Artmed; 2007.

13. Gil AC. Como elaborar projetos de pesquisa. $4^{\mathrm{a}}$ ed. São Paulo: Atlas; 2002.
14. Cañete I. Humanização: desafio da empresa moderna. Porto Alegre: Artes e Ofícios; 1996.

15. Mendes RA. Ginástica Laboral (GL): Implantação e Benefícios nas Indústrias da Cidade Industrial de Curitiba [dissertação de Mestrado]. Curitiba: Centro Federal de Educação Tecnológica do Paraná; 2000.

16. Silveira MG. Efeitos da ginástica laboral nas variáveis morfológicas, funcionais, estilo de vida e absenteismo dos trabalhadores da indústria farmacêutica de Montes Claros - MG [dissertação de Mestrado]. Vila Real: Universidade de Trásos-Montes e Alto Douro; 2008.

17. Reis ES. Análise ergonômica do trabalho associada à cinesioterapia de pausa como medidas preventivas e terapêuticas às em um abatedouro de aves [dissertação de Mestrado] Florianópolis: UFSC; 2001.

18. Martinez JFN, Matiello Júnior E. Os limites da ginástica laboral para compreensão dos determinantes de saúde de trabalhadores bancários. Pensar a Prática. 2012; 15(3):610-624.

19. Coelho CF, Burini RC. Atividade fisica para prevenção e tratamento das doenças crônicas não transmissiveis e da incapacidade funcional. Rev Nutr (Campinas).2009; 22(6):937-946.

20. Bielemann $R$, Knuth AG, Hallal PC. Atividade fisica e redução de custos por doenças crônicas ao Sistema Único de Saúde. Rev Bras Ativ Fis Saúde. 2010;15(1):9-14.

21. Reis $P$, Moro ARP, Contijo LA. A importância da manutenção de bons niveis de flexibilidade nos trabalhadores que executam suas atividades laborais sentados. Revista Produção Online. 2003;3(3):00-16.

22. Candotti $C T$, Stroschein $R$, Noll M. Efeitos da ginástica laboral na dor nas costas e nos hábitos posturais adotados no ambiente de trabalho. Rev Bras Ciênc Esporte. 2011;33(3):699-714.

23. Alencar MCB, Ota NH. 0 afastamento do trabalho. Rev Ter Ocup Univ São Paulo. 2011; 22(1):60-67.

24. Picoloto $D$, Silveira E. Prevalência de sintomas osteomusculares e fatores associados em trabalhadores de uma indústria metalúrgica de Canoas - RS. Ciênc saúde coletiva. 2008;12(2):507-516.

25. Szeto GPY, Lam P. Work-related musculoskeletal disorders in urban bus drivers of Hong Kong. J Occup Rehabil. 2007;17(2): 181-198. 